\title{
Gemeinschaftsnarrative unter Kindern und Jugendlichen in marginalisierten Quartieren
}

\author{
Strategien im Umgang mit stigmatisierenden Diskursen am Mehringplatz
} in Berlin-Kreuzberg

Nils Zimmer

\begin{abstract}
Das Quartier am Mehringlatz in Berlin-Kreuzberg ist seit vielen Jahren einer immensen Stigmatisierung ausgesetzt. Die Diskurse rund um den Stadtteil zeichnen sich durch eine (Re-)Produktion rassistischer und diskriminierender Bilder aus. Diese ignorieren soziale Ungleichheiten und strukturelle Benachteiligungen infolge gesellschaftlicher Machtverhältnisse. Vor allem die Kinder und Jugendlichen aus dem Stadtteil werden dabei eigenen Repräsentationen beraubt. Daher bleiben ihnen kaum Möglichkeiten, die vorgefertigten Bilder und die ihr innewohnende Gewalt aufzubrechen. Der vorliegende Beitrag geht der Frage nach, welche Strategien die Kinder und Jugendlichen im Umgang mit diesen Darstellungen des Quartiers entwickeln und wie sie diesen für sie so wichtigen Raum selbst wahrnehmen. Der Artikel basiert auf den Ergebnissen einer ethnographischen Studie.
\end{abstract}

Ersteinreichung: 30. November 2020; Veröffentlichung online: 26. November 2021

An English abstract can be found at the end of the document.

\section{Einleitung}

Anfang 2016 begann ich als sozialpädagogischer Betreuer im Offenen Bereich einer Kinder- und J ugendeinrichtungim Quartier am Mehringplatz in Berlin-Kreuzberg zu arbeiten - in einer Gegend, die keinen guten Ruf genießt. Das Quartier zeichnet sich durch einen hohen Anteil an Familien mit Migrationsgeschichte sowie überdurchschnittlich viele Haushalte in ökonomisch prekären Verhältnissen aus. DasführtfürvieleBewohner_innen zu einer Überschneidung multipler benachteiligender Faktoren, zu denen auch die Stigmatisierung des Viertels beiträgt. Dieser Beitrag wirft eingangs einen Blick auf diesozialräumlichen Strukturen des Stadtteils. Anschließend gehtesum dieFrage, welcheKräftean den stigmatisierenden Zuschreibungen des Quartiers und seiner Bewohner_innen beteiligt sind. Das macht eine Auseinandersetzung mit den Darstellungen des Quartiersmanagements sowie mit der raumbezogenen Stadtpolitik des Programms „Stadtteile mit besonderem Entwicklungsbedarf - Soziale Stadt“ (kurz: „Soziale Stadt") erforderlich. Darauf folgt eine Betrachtung des medialen Umgangs mit der Gegend, bevor der Beitrag wissenschaftliche Perspektiven auf marginalisierte Stadtteile und deren Stigmatisierung diskutiert. Da es letztlich die Erzählungen der Kinder und J ugendlichen waren, diemich auf die Idee brachten, diese Untersuchung durchzuführen, geht der Beitrag nach einer kurzen 
Erläuterung des methodischen Vorgehens und einer Reflexion meiner eigenen Rolle im Feld der zentralen Fragenach: Welche Strategien entwickeln diese Kinder und J ugendlichen im Umgang mit den stigmatisierenden Zuschreibungen? Dabei lassen sich drei zentrale Narrative ausmachen, die sich grob wie folgt charakterisieren lassen: erstens eine diskursive Umkehr gesamtgesellschaftlicher Mehrheits- und Machtverhältnisse, zweitens positive (Gegen-)Erzählungen und drittens Formen der Aneignung des Stigmas. Alle drei Narrative bedürfen hinsichtlich ihrer Verwobenheit mit multiplen Ausgrenzungs- und Diskriminierungserfahrungen eines genauen analytischen Blicks.

\section{Das Quartier am Mehringplatz}

Das Quartier am Mehringplatz liegt im nordwestlichen Teil Kreuzbergs und damit im Zentrum Berlins. Architektonisch zeichnet es sich durch Gebäude im Stil des sozialen Wohnungsbaus der 1970erJ ahre und großeWohnblocks aus. Laut dem Amt für Statistik Berlin-Brandenburg haben knapp 71 Prozent aller Einwohner_innen einen sogenannten Migrationshintergrund. Viele Haushalte leben in ökonomisch prekären Verhältnissen. Gemessen am Altersdurchschnitt der Bewohner_innen handelt es sich um einen vergleichsweisejungen Stadtteil (vgl. QM2017: 7). Das Bild des Quartiers ist seit etlichen Jahren von großflächigen Bauarbeiten geprägt, in deren Folge Teile des Gebiets immer wieder abgesperrt sind. Regelmäßigbetonen die aus dem Quartier stammenden Kinder und J ugendlichen, wie sehr sie sich dadurch räumlich eingeschränkt fühlten - manchevon ihnen kennen die Gegend gar nicht anders. Nicht selten mutmaßen sie, die Arbeiten würden nur deshalb so lange anhalten, weil sich schlichtweg niemand für die Gegend und deren Bewohner_innen interessiere.

Seit 2005 ist das Quartier Teil des Programms „Soziale Stadt“. Bauliche Mängel, fehlendeRessourcenim Bereich kulturellerund sozialer Angebote, die prekäre wirtschaftliche Lage eines Großteils der Haushalte sowie mangelnde Möglichkeiten politischer Partizipation fürvieleBewohner_innen aufgrund der fehlenden deutschen Staatsangehörigkeitführen dazu, dass das Quartier als marginalisiert bezeichnet werden kann (vgl. Ottersbach 2003: 34 f.).

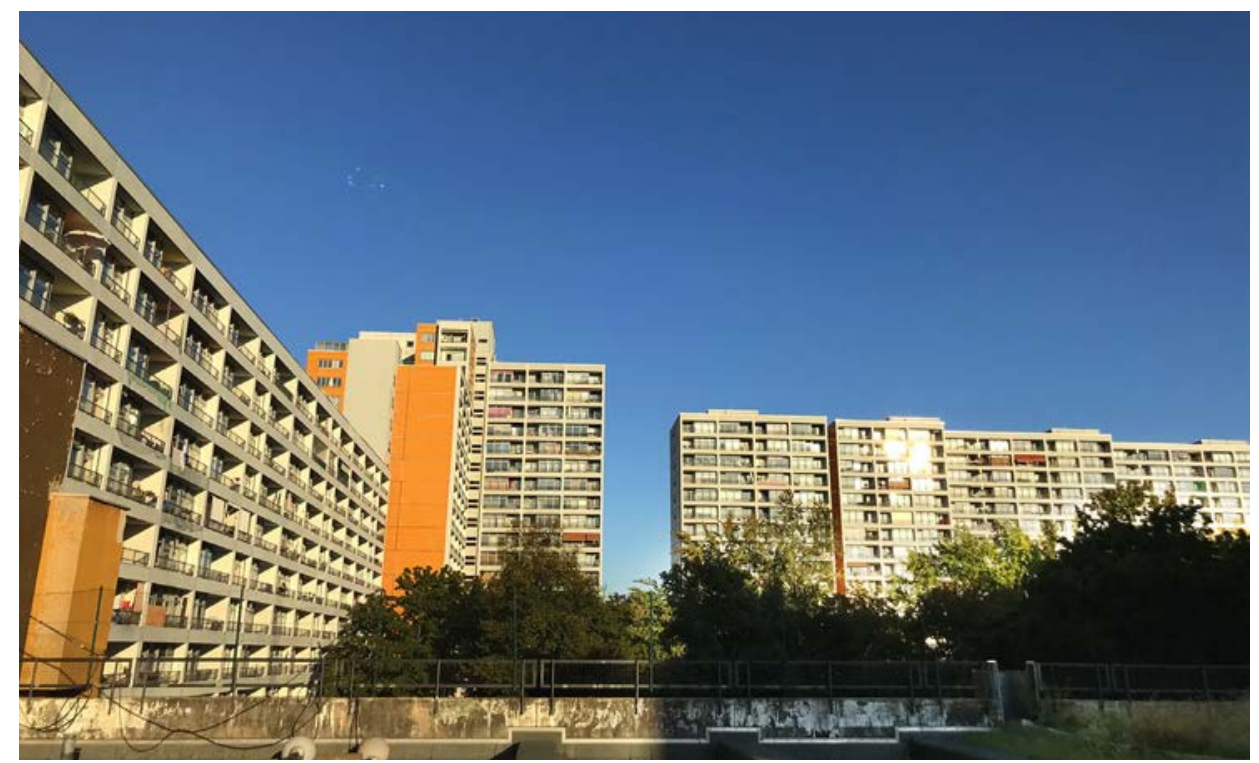

Abb. 1 Hochhäuser im Quartier am Mehringplatz (Quelle: eigene Aufnahme) 
Abb. 2 Seit Jahren ist der Mehringplatz wegen Bauarbeiten gesperrt (Quelle: eigene Aufnahme)

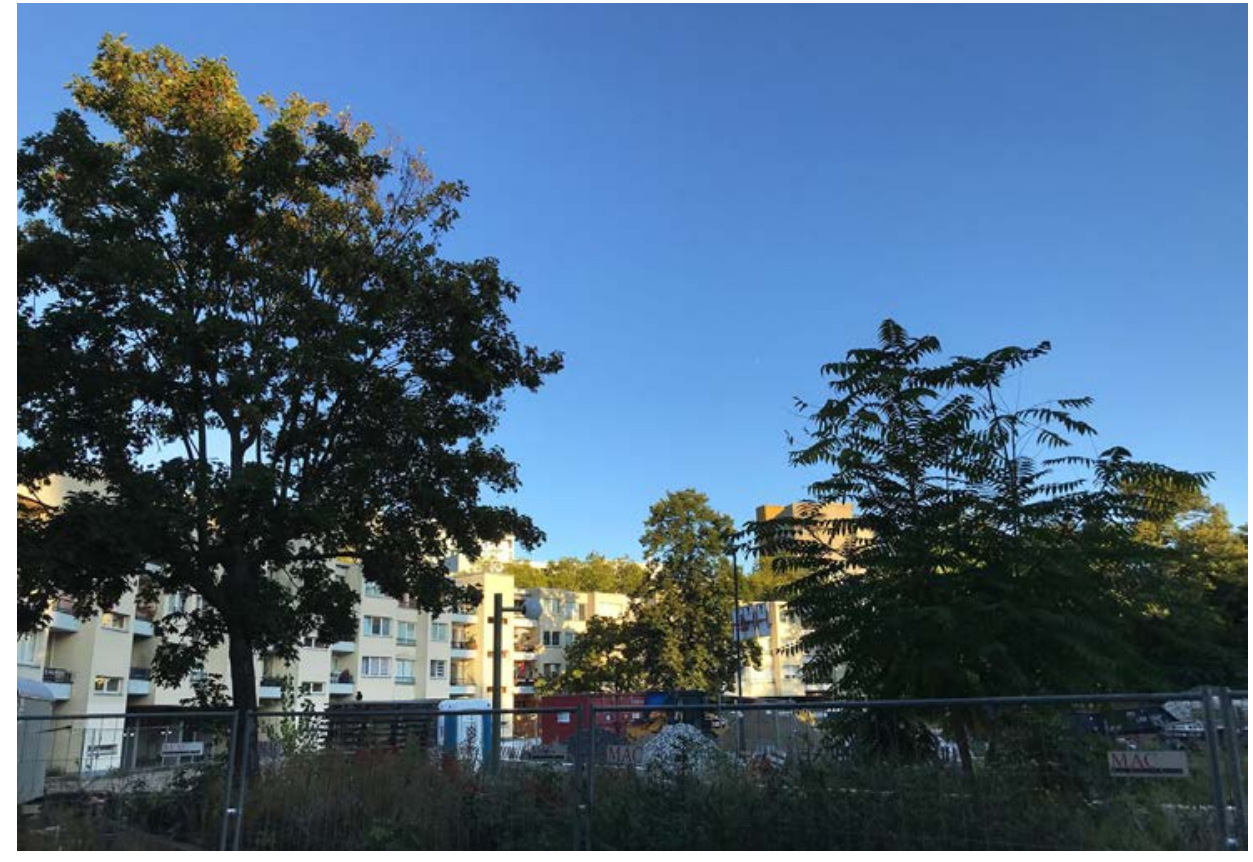

Solche Stadtteile, die sich durch vielfältige benachteiligende Faktoren auszeichnen, sind ein Ausdruck kapitalistischer Urbanisierungsprozesse (vgl. Harvey 2009 [1973]: 110 ff.).[1] Diese Prozesse führen dazu, dass sich gesellschaftliche Ungleichheitsverhältnisse in den städtischen Strukturen abbilden (vgl. Bürkner 2020: 158; Kemper 2018; Alisch 2018: 504 f.; Farwick 2007: 114 f.). Dahinter verbergen sich Strategien, die aus raumanalytischer Sicht als Territorialisierung bezeichnet werden können und eine spezifische Form der Machtausübung ermöglichen (vgl. Belina 2017: $88 \mathrm{ff}$.). Hans-J oachim Bürkner (2020) bemerkt dazu: „Segregation entstehtnichtnur in der sozialen Praxis, sie wird auch politisch inszeniert, d.h. je nach Interessenlage gefördert, bekämptt oder hingenommen“ (Bürkner 2020: 160 f.). Betroffene Stadtviertel weisen eine tendenzielle Homogenität hinsichtlich der Klassenlage ihrer Bewohner_innen auf bei gleichzeitig deutlicher Heterogenität hinsichtlich aller anderen Faktoren (vgl. Alisch 2018: 505). Dabei fallen ethnische und soziale Segregationsprozesse häufig ineinander, da migrantische Familien überproportional stark von prekären Beschäftigungsverhältnissen betroffen sind (vgl. ebd.: 507; Farwick 2018: 5).[2] Das darf jedoch nicht dazu führen, „soziale Problemlagen einseitig auf die Dimension Ethnie zu reduzieren“ (Alisch 2018: 507) oder „falsche Schlüsse hinsichtlich einer mangelnden Integration der Zugewanderten“ (ebd.) zu ziehen. Zudem hat die Marginalisierung des Quartiers keineswegs nur sozioökonomische Faktoren. Auch Aspekte von Stigmatisierung müssen in den Blick genommen werden (vgl. Wacquant/ Slater/ Borges Pereira 2014: 1272; Ottersbach 2009: 55 f.; Gebhardt 2001: 12), denn ein negatives Image „fördert und festigt schließlich den Prozess der Marginalisierung" (Chamakalayil et al. 2017: 179) und ist so an der (Re-)Produktion beziehungsweise Verstärkung sozialer Ungleichheiten beteiligt (vgl. Bürkner 2020: 160; Chamakalayil et al. 2017: 178 f.; Hill 2016: 55; Ottersbach 2009: 55 f.; Wacquant 2007: 400). Da es in erster Linie die mediale und stadtpolitische Berichterstattung ist, die zur Konstruktion des Stadtteils als Problemquartier beiträgt, unterziehe ich diese im Folgenden einer genaueren Analyse. 


\section{Die Konstruktion des Stadtraums als benachteiligtes Quartier}

Für das Quartier am Mehringplatz wird seit 2013 alle zwei J ahre das Integrierte Handlungs- und Entwicklungskonzept (IHEK) veröffentlicht. Es beschreibt aktuelleEntwicklungen und vermeintliche Problemedes Gebiets und leitet daraus Handlungsbedarfe sowie Zielvorstellungen ab. Den Handlungskonzepten wird seitens der sozialen Stadtpolitik eine hohe Signifikanz eingeräumt (vgl. Walther/ Güntner 2007: 396 f.). Im Folgenden werfe ich einen genaueren Blick auf das vom Quartiersmanagement am Mehringplatz veröffentlichte IHEK für die Jahre 2017 bis 2019. Dieses nimmt eine konstante Dichotomisierung zwischen autochthonen Deutschen und migrantischen Familien vor. Letzteren werden vehement desintegrative Eigenschaften und ein geringes Verantwortungsbewusstsein für den Sozialraum unterstellt. Neben der insgesamt starken defizitorientierten Fokussierung auf die migrantischen Familien des Stadtteils macht der Bericht Müll und Schmutz, ein unattraktives Erscheinungsbild, drogen- und alkoholkonsumierende Gruppen, diegeringe Kaufkraft der Bewohner_innen, hoheErwerbslosigkeit, einen insgesamt niedrigen Bildungsstand und kriminelle J ugendliche als Probleme des Quartiers ausfindig (vgl. QM 2017). Mit Blick auf Letztere heißt es etwa, dass Ansammlungen von J ugendlichen in den Abendstunden das Unsicherheitsgefühl im Quartier förderten (vgl. ebd.: 17). Zugleich führen Bautätigkeiten und Modernisierungsarbeiten sowieausgelaufene Mietpreisbindungen im Gebiet zu immensen Mietsteigerungen und zur Entstehung von (Eigentums-)Wohnungen im hochpreisigen Segment (vgl. ebd.: 16). Dies mache „Prognosen über die soziale Schicht der neuen Bewohnerschaft möglich“ (ebd.: 16), deren Einbindung für das Quartiersmanagement von fundamentaler Bedeutung ist. Um diese Einbindung zu erreichen, sollen Bildungseinrichtungen, die sich seit Jahren in Sanierung befinden, endlich fertiggestellt und so neu ausgerichtet werden, dass sie nicht mehr nurvon der bisherigen Bewohner_innenschaft genutztwerden (vgl. ebd.: 19). DieseMaßnahmen betrachtet das Quartiersmanagement als entscheidenden Schritt, „um die weitere Ausbildung von Parallelgesellschaften am Mehringplatz zu verringern" (ebd.: 5). Bei den Kindern und J ugendlichen, um die es in der vorliegenden Untersuchung geht, sorgt die Entstehung eben jener neuen Nachbarschaften hingegen für wenig Begeisterung. Der 20-jährige Deniz, der am Mehringplatz aufgewachsen ist und noch immer dort lebt, äußert sich dazu in einem kürzlich ausgestrahlten Bericht des Rundfunks BerlinBrandenburg (rbb 2021) folgendermaßen: „Für mich persönlich ist es eine Sorge, weil man fühlt sich irgendwie so, dass man hier außen rum zugebaut wird von neuen Leuten. Und dass wir irgendwann alle hier innen drin rausgenommen werden. So fühlt sich das an für uns" (rbb 2021).

Das Quartiersmanagement als Herausgeberin des IHEK 2017-2019 ist Teil einer Interventionsstrategie, deren Ziel die Stabilisierung defizitärer Nachbarschaften ist. Diese sollen zu „,selbstständig lebensfähigen Stadtteilen mit positiver Zukunftsperspektive“ (Difu 2003: 299) gemacht werden. Als eigenständiger Maßnahmenschwerpunkt wurde 2005 die „Integration von Migrantinnen und Migranten“(Reimann/Schuleri-Hartje2005) hinzugefuigt. So bringt das Programm „Soziale Stadt“ betreffende Stadtteile nicht nur mit 
fehlendem ökonomischem Kapital, sondern stets auch mit Migration in Verbindung und problematisiert diese (vgl. Weber 2013; Lanz 2002: 69 ff.). Das hat eine (Re-)Produktion gesellschaftlicher Differenzierungen zur Folge und macht insbesondere Menschen mit Migrationsgeschichte zu Träger_ innen von Problemen oder zu Abweichler_innen (vgl. Weber 2013). Dieser Hergangkann als Prozess der Entgleichung bezeichnet werden (vgl. dazu Lanz 2007: 357 ff.; Terkessidis 2004: 195 ff.). Thomas Franke (2014) weist auf die Schwierigkeiten der Bewertung vermeintlicher Defizite hin, die sich nicht nur auf Statistiken berufen, sondern ebenso auf subjektive Aussagen über den sozialen Zusammenhalt oder Gefühle der (Un-)Sicherheit innerhalb eines Quartiers (vgl. ebd.: 162). Im FalledesMehringplatzesstütztsich dasQuartiersmanagement dabei nicht unwesentlich auf Informationen des Quartiersrats, der sich aus Anwohner_innen und lokalen Akteur_innen zusammensetzt. In diesem Quartiersrat sind jedoch Personen, dienichtautochthon deutsch sind, deutlich unterrepräsentiert. Die Perspektive von Kindern und J ugendlichen fehlt gänzlich. Das ist für solche Formen der quartiersbezogenen Beteiligung nicht ungewöhnlich (vgl. Holm 2010). Margit Mayer (2003) schreibt dazu: „In Gebieten wo das Quartiersmanagement zum Einsatz kommt, ist ein häufig beobachteter Effekt, dass bestimmte Stadtteilbewohnergruppen beteiligt werden bei gleichzeitiger Ausgrenzung anderer (unerwünschter oder problematischer) Gruppen“ (ebd.: 272). Diese Einschätzung deckt sich mit derWahrnehmungmehrererJ ugendlicher, diemirgegenüber einst äußerten, deshalb keine Lust zu haben, sich am Quartiersrat zu beteiligen.

Als wichtigen Schritt betrachtet das Quartiersmanagement die Schaffung einer „sozialen Mischung", womitim Wesentlichen derZuzugsozioökonomisch bessergestellter, autochthon deutscher Familien und deren Einbindungin das Quartier gemeint ist. Solche Formen der Durchmischung sollen bewirken, dass Quartiere wiedasam Mehringplatz „,normalisiert “ werden. Dadurch wird allerdingsnichtnur eineVerdrängungökonomisch deprivilegierter Haushalte forciert (vgl. Böhmer/Holm/Jacob 2021: 136 ff.; Rinn 2018: 21 ff.; Holm 2010). Obendrein wird die fehlende „soziale Mischung“ nur dann problematisiert, wenn es sich um Quartiere handelt, dievon Migration und Armut geprägt sind (vgl. Ha/ Schneider 2020: 62; Alisch 2018: 509 f.; Ronneberger/ Tsianos 2009: 144). MoritzRinn (2018) kommt folglich zu dem Schluss: „Diese Arbeit an derVerbürgerlichung der Stadt will soziale Ungleichheiten nicht abbauen, sondern re-arrangieren " (ebd.: 22). Es verwundert daher kaum, dass eine solche (Stadt-)Politik im Kampf gegen Armut wirkungslos ist und auch dieräumlicheKonzentration prekärer Haushalteinnerhalbstädtischer Gebiete nicht abgenommen hat (vgl. Zimmer-Hegmann 2014: 126 f.; Ronneberger/ Tsianos 2009: 144 f.). Stattdessen führt dieLokalisierungsozialer Problemeim Sinneeiner räumlichen Verortung dazu, bestimmteStadtteileals benachteiligt zu klassifizieren und damit aktiv zu deren Stigmatisierung beizutragen (vgl. Preissing 2019a: 45f.; Weber 2013: 268; Walther/ Güntner 2007: 395).

\section{4. ,...der größte Schandfleck der Hauptstadt“ - mediale Darstellungen}

In verschiedenen Berliner Tageszeitungen fanden sich in den vergangenen J ahren Berichte über das Quartier am Mehringplatz, die sich einer sehr 
bildgewaltigen Sprache bedienen und regelmäßig völlig überzogene Schreckensszenarien kreierten. Auch dieseZeitungsberichteverweisen meist ohne ersichtlichen Grund auf den hohen Anteil migrantischer Familien oder die vergleichsweise hohe Anzahl von Personen, die Transferleistungen beziehen. Vorallem diejugendlichen Bewohner_innen des Quartiers werden wiederkehrend mit Kriminalität, Drogenhandel und/ oder Gewalt in Verbindung gebracht.[3] In mehreren Berichten über das Quartier am Mehringplatz ist außerdem von einer Ghettoisierung des Stadtteils die Rede. Die Beschreibung des Quartiers als dreckig, vermüllt oder hässlich zieht sich dabei wieein roter Faden durch diverse Berichte. So sehr sich dieArtikel auch vordergründigunterscheiden, setzen siedoch allesamt auf die Konstruktion eines Angstraumes und machen sich dadurch automatisch stark für eine Stadtpolitik, dievon Sicherheit, Ordnung und Sauberkeit geprägt ist. Dass auch die J üngeren aus dem Quartier sehr genau um diese Darstellungen wissen, wurde mir bewusst, als mir ein damals neunjähriger J unge auf der Straße entgegenkam und mir zurief: „Wir sind in der Zeitung! “Wenig später verstand ich was er meinte. Etliche der Kinder und J ugendlichen hatten sich per Handy gegenseitig Screenshots eines Artikels aus der B.Z. vom 20. August 2018 zugeschickt und diese auf Instagram geteilt. Die Überschrift dieses Artikels lautete: „Der Mehringplatz ist der größte Schandfleck der Hauptstadt“" (Wilkens/ Klier 2018).

Dramatisierenden Darstellungen über das Leben in marginalisierten Quartieren liegt vielfach eine ethnisierende Sichtweise zugrunde. Die vielfältigen Lebensentwürfe der Bewohner_innen solcher Stadtteile werden ignoriert und ihregesellschaftlichen Entstehungsbedingungen ausgeblendet (vgl. Geisen/Riegel/Yildiz 2017: 4 f.). Stattdessen werden sozialer Zerfall und Desorganisation zu inhärenten Eigenschaften der Viertel erklärt (vgl. Wacquant/ Slater/ Borges Pereira 2014: 1274). Damit wird Raum zum „distinctive anchor of social discredit“ (ebd.: 1272). Dirk Gebhardt (2001) spricht in diesem Zusammenhang von einer Pathologisierung städtischer Orte (vgl. ebd.: 28), die letzten Endes zu einer „räumliche[n] Konstruktion des Anderen“ (Lanz 2007: 146) führt.

Zwar existieren auch Presseberichte, die andere Aspekte des Quartiers hervorheben, die negativen Darstellungen können aber durchaus als

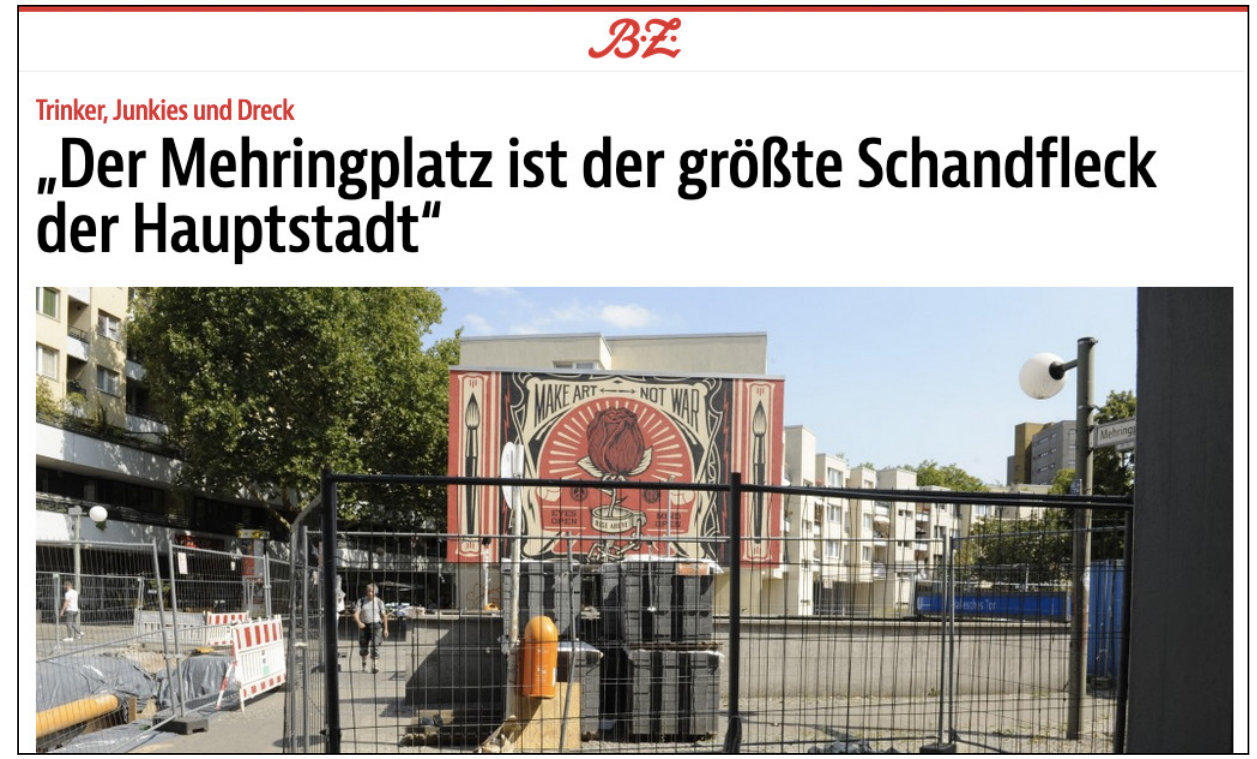

Abb. 3 Screenshot B.Z. (Quelle: Wilkens/ Klier 2018) 
hegemonial bezeichnet werden. Dabei dienen medial erzeugte Bilder nie ausschließlich einer Beschreibung der Realität, sondern immer auch deren Konstruktion (vgl. Yildiz 2016: 31 ff.; Schulze/ Spindler 2006: 65; Gebhardt 2001). Dirk Gebhardt macht diesbezüglich „Ideologie, Hegemonie und common sense" (Gebhardt 2001: 12, Hervorhebung im Original) als Mechanismen aus, „die bestimmten Wahrheiten zur Durchsetzung verhelfen“ (ebd.). Bernd Belina verweist hierzu auf das Prinzip der Abstraktion, das ein entscheidendes Merkmal des „strategischen Einsatzes von Raumideologien" (Belina 2006: 103) sei und das auch genutzt werde, um ganze Personengruppen zu stigmatisieren (vgl. ebd.: 114 ff.). An solchen Konstruktionen sind allerdings keineswegs nur Medien und (Stadt-)Politik beteiligt, sondern auch die Wissenschaft.

\section{Territoriale Stigmatisierung}

Loïc Wacquant fasst die Stigmatisierung städtischer Räume unter dem Begriff der "territorialen Stigmatisierung“ zusammen. Dazu schreibt er: „Territorial stigmatization [...] is [...] a novel urban phenomenon characteristic of the post-Fordist metropolis. " (Wacquant/ Slater/ Borges Pereira 2014: 1275). Auch in sozialwissenschaftlichen Debatten werden städtische Räumeimmer wieder als „,soziale Brennpunkte“ markiert und als besonders konfliktgeladen und defizitär dargestellt. Dabei handelt es sich um ein „für die deutscheStadtforschung charakteristische[s] Bild von räumlicher Segregation, das Stadt dichotom zwischen ,ethnischen Kolonien' und ,Ghettos' und bürgerlich ,normalen 'Sozialräumen der Mehrheitsgesellschaft spaltet“ (Lanz 2007: 247). Nach wievor kann (institutioneller) Rassismus innerhalb der deutschen Stadtforschung als blinder Fleck bezeichnet werden (vgl. Ronneberger/Tsianos 2009: 148 f.; Lanz 2007: 173). Dabei sind es häufig staatlich geförderte Auftragsforschungen, diesich reduktionistischer Erklärungsmuster bedienen, um vermeintlicheRisiken von Migration und Urbanisierung zu behaupten (vgl. dazu Mecheril/ Polat 2019).

„Aus solchen falschen und interessengeleiteten Abstraktionen können ganze ideologische Theoriegebäude entstehen, die sich in den Sozialwissenschaften in großer Zahl finden. Gemeinsam ist ihnen, dass in ihren Basisannahmen bereits das Interesse und die Praxis stecken, die sie legitimieren sollen." (Belina 2006: 104)

Auf diese Weise tragen „,wissenschaftliche Diskussionen [...] mit zur Marginalisierung von Stadtvierteln bei“ (Hill 2016: 54).

Die Folgen dieser Stigmatisierung für die Bewohner_innen der betroffenen Viertel sind vielfältig. Zu ihnen zählen Adressendiskriminierung, im Falle migrantisch geprägter Stadtteile die Rassifizierung etlicher Anwohner_innen (vgl. Kirkness/Tijé-Dra 2017: 2), behördliche Willkür sowie besonders repressive Formen des Polizierens (vgl. Belina 2017; Wacquant/ Slater/ Borges Pereira 2014: 1275; Wacquant 2018 [2008]). In früheren Publikationen weist Loïc Wacquant zusätzlich auf den Wegfall kollektiver Gemeinschaft und im Zuge dessen auf Distanzierungen der Bewohner_innen von ihrem Stadtteil hin (vgl. etwa Wacquant 2006: 29). Er spricht in diesem Kontext von einer „Auflösung des ,Ortes “" (vgl. Wacquant 2007; Wacquant 2018 [2008]).[4] Gerade 
diese Annahme blieb nicht unwidersprochen (vgl. exemplarisch Kirkness 2014; Slater/ Anderson 2012). Dabei bezieht sich die Kritik an seinem Konzept vor allem auf die vermeintliche Passivität, die den Betroffenen unterstellt wird (vgl. dazu auch Glasze/ Pütz/Tijé-Dra 2014: 59f.).[5] Auch in meiner Untersuchung wird deutlich, dass die Distanzierung der Kinder und J ugendlichen von ihrem Viertel nur einevon vielen möglichen Reaktionen ist und dass diesekeineswegs mit dem Verlust positiver Gefühle zum Quartier einhergehen muss.

In diesem Sinne ergänzten Wacquant/ Slater/ Borges Pereira (2014) in einem jüngeren Beitrag: „even as some strive to disregard or to resist spatial stigma" (ebd.: 1275). Aufbauend auf dieser Annahmenehmen sieeine Differenzierung verschiedener Strategien im Umgang mit territorialer Stigmatisierungvor, diegrobin „Submission“ (ebd.: 1276) und „Recalcitranceto resistance" (ebd.) unterteilt werden können. Anhand eines Beispiels bemerken sie: „The seemingly incongruent mating of strategies [...] is stabilized by strictly segregating the scenes on which each is deployed." (Ebd.) Dennoch weisen Paul Kirkness und Andreas Tijé-Dra (2017) zu Recht darauf hin, Wacquant habe „not devoted much writing to the contestations of territorial stigmatization" (ebd.: 3). Folglich müssen Wacquants Arbeiten um eine Reihe wissenschaftlicher Beiträge ergänzt werden, die sich nicht nur der vielschichtigen Prozesse territorialer Stigmatisierung fernab reduktionistischerErklärungsmuster annehmen, sondern diesezudem um dievielfältigen Umgangsweisen der Bewohner_innen mit stigmatisierenden Diskursen erweitern (vgl. exemplarisch die Arbeiten von Preissing 2019a und Yildiz 2016, den Sammelband von Kirkness/Tijé-Dra 2017 sowie die Themenhefte von Wacquant/ Slater/ Borges Pereira 2014 und Glasze/ Pütz/ Tijé-Dra 2014).[6] Entsprechende Veröffentlichungen gilt es in Bezug auf den vorliegenden Untersuchungsgegenstand um Arbeiten zu ergänzen, die sich mit aktuellen Problemlagen und gesellschaftspolitischen sowiepolarisierenden Diskursen rund um die Themen Kindheit und J ugend auseinandersetzen, insbesondere solcher, die sich im Hinblickauf die Situation von Kindern und J ugendlichen der zweiten und dritten Migrationsgeneration transdisziplinärer Ansätze bedienen und dabei gesellschaftliche Ungleichheitsverhältnisse berücksichtigen (vgl. exemplarisch die Beiträge aus Geisen/Riegel/Yildiz 2017; Groenemeyer/Hoffmann 2014; Riegel/Geisen 2010).

Kritische (Stadt-)Forscher_innen nehmen sich seit geraumer Zeit einer dekonstruktivistischen Lesart an, um die ich mich hier ebenfalls bemühe. Vor dem Hintergrund, dass den Kindern und J ugendlichen des Quartiers eigene Repräsentationen im öffentlichen Diskurs verwehrt bleiben, gilt mein Interesse der Frage, wie sie das Quartier entgegen hegemonialer Diskurse wahrnehmen, welche Darstellungen sie im Zuge dessen vornehmen und welche Strategien sich dabei im Umgang mit dessen Stigmatisierung ausmachen lassen. Parallelen zu den Beobachtungen anderer Untersuchungen sind unverkennbar (vgl. unter anderem Preissing 2019a; Yildiz 2016; Kirkness 2014; Schulze 2010).

\section{Methodisches Vorgehen}

Ausgangspunkt für meine Untersuchung ist eine Perspektive, die Kinder und Jugendliche als Expert_innen ihres Alltags ernst nimmt und auf ihr 
Wissen fokussiert. Die Kinder und J ugendlichen sind aktiv Handelnde. Sie entwickeln eigene Strategien, deren kritische Reflexion Ziel dieses Beitrags ist. Methodisch eignen sich dazu ethnographische Ansätze wie die teilnehmende Beobachtung besonders gut (vgl. Heinzel/ Kränzl-Nagl/ Mierendorff 2012: 18 ff.). Das bedeutet zugleich, „immer auch anzuerkennen, dass zwischen Kindern und Erwachsenen eine Perspektivendifferenz besteht“ (ebd.: 17). Im Falle meiner Untersuchung muss diese zusätzlich um die Reflexion eigener Normalitätsvorstellungen und der damit verbundenen (Re-)Produktion von Differenzkategorien erweitert werden (vgl. Preissing 2019b: 145 f.; Mecheril/Polat 2019: 52; Sylla et al. 2019: 91f.).

Mein Zugang zum Feld, mein Verhältnis zu den Kindern und J ugendlichen und dieFrage, wieich von ihnen wahrgenommen werde, sind dabei von grundlegender Bedeutung. Über einen Zeitraum von knapp vier J ahren war ich als sozialpädagogischer Betreuer im Offenen Bereich eines anerkannten freien Trägers der Kinder- und J ugendhilfe im Quartier am Mehringplatz tätig. Dadurch nahm ich mehrere Tage pro Woche am Alltagsgeschehen rund um den Mehringplatz teil. Der vorliegende Beitrag baut im Kern auf den Ergebnissen meiner Masterarbeit auf. Das empirische Material stammt aus Beobachtungen, die ich während meines Arbeitsalltags machte. Mein Aufenthalt im Feld reichte nicht nur weit über die bloße Forschung hinaus. Diese vermischte sich mit meiner beruflichen Tätigkeit und emotionalen Bezügen, was eine kritische Reflexion erforderlich macht. Als sozialpädagogischer Betreuer war ich in ständiger Interaktion mit den Kindern und J ugendlichen, weshalb meine Rolle aus Forscher_innenperspektive als „aktiv-teilnehmend“ (Atteslander 2010: 95) bezeichnet werden kann. Die Ambivalenz dieser Rolle machte sich auf zweierlei Weise bemerkbar: Zum einen war es mir aufgrund meiner beruflichen Tätigkeit nicht möglich, mich über längere Zeit aus dem Feld zurückzuziehen. Zum anderen entwickelte ich zu den Kindern und J ugendlichen ein Verhältnis, dass durch Vertrauen und Loyalität gekennzeichnet ist. Das muss jedoch nicht zwangsläufig zum Verlust einer Sichtweise führen, wie es sie zum Zwecke der Analyse zu bewahren gilt (vgl. dazu Flick 2017: 291 ff.; Atteslander 2010: 94 f.). Stattdessen bedarf es einer der Situation angepassten Distanzierung und Reflexion. Diese bestand aus einem wiederkehrenden Wechsel zwischen Analyse und Datengewinnung sowie der fortwährenden Produktion von Text (vgl. Breidenstein et al. 2015: 109 ff.). Eine bedeutende Rolle spielte während desForschungsprozesses dieFokussierungaufmein Forschungsinteresse. Ich ließ mich diesbezüglich von dem leiten, was den Kindern und J ugendlichen in ihren Erzählungen augenscheinlich von Bedeutungwar (vgl. dazu ebd.: 88 f.). Hinsichtlich der Nachvollziehbarkeit der gesammelten Daten erwies sich umfassendes Hintergrund- und Kontextwissen als unabdingbar (vgl. ebd.: 114 ff.). Obwohl ich die Kinder und Jugendlichen, mit denen ich zu dieser Zeitregelmäßigarbeitete, überdas Vorhaben meiner Masterarbeit informierte und ihnen gegenüber darlegte, welches Ziel ich damit verfolge, stand dies nie im Mittelpunkt unserer Beziehung. Vielmehr schien es so, alssei dieses bei den meisten schnell wieder in Vergessenheit geraten. Bei der Anfertigung dieses Artikels sprach ich erneut mit ihnen und fragte sie, ob diese Veröffentlichung für sie in Ordnung sei, ob sie Bedenken hätten und bot ihnen an, gemeinsam über den Beitrag zu sprechen (siehe dazu DGSA 2020: 6 ff.). Im Zuge dessen 
sicherte ich ihnen zu, sämtliches Material so zu anonymisieren, dass eine Identifikation durch Außenstehende unmöglich sei.[7]

\section{Ergebnisse}

DieStrategien, diedie Kinder und J ugendlichen im Umgangmit den hegemonialen Diskursen über den Stadtteil entwickeln, fallen sehr unterschiedlich aus. Dabei lassen sich drei zentraleNarrativenachzeichnen: Erstens kehren die Kinder und J ugendlichen die gesellschaftlichen Mehrheits- und Machtverhältnissediskursivum. Zweitensnehmen sie positiv Bezugauf das Leben im Stadtteil und kreieren dadurch (Gegen-)Erzählungen und drittens eignen sie sich das Stigma an. Im Folgenden präsentiere ich einige Schlaglichter meiner Untersuchungsergebnisse.

\subsection{Diskursive Umkehr gesellschaftlicher Mehrheits-} und Machtverhältnisse

Ein anhaltendes Prinzip der Kinder und J ugendlichen ist die Markierung von Personen, die sie als nicht zugehörig zum Quartier wahrnehmen. Das können Besucher_innen, Tourist_innen oder andere Personen sein, die sie nicht kennen. Der gleichen Logik folgt die wiederkehrende Benennung von Personen, die sie als „Deutsche“ lesen. Sie bringen dabei wiederholt zum Ausdruck, dass sich autochthone Deutsche gemäß ihrer Vorstellungen am Mehringplatz zu integrieren hätten. Solche Benennungspraxen stehen in unmittelbarem Bezug zu ständigen Verweisen der Kinder undJ ugendlichen, dass das Quartier eine „Ausländergegend“ oder „Kanakengegend“ sei. Einerseits heben sie immer wieder hervor, dass es Regeln im Stadtteil gäbe, an die es sich zu halten gelte. Andererseits lassen sie kaum eine Gelegenheit aus, um zu betonen, dass es sich um ihr Viertel handle, in dem sie tun und lassen könnten, was siewollten. In solchen Momenten spiegeln sieauf überzeichnete Weise wider, was sie aus den öffentlichen Diskursen kennen nämlich dieständige und zumeist völlig unvermittelte Bezugnahmeauf den hohen Anteil an Personen im Gebiet, dienicht autochthon deutsch sind, und damit verbundene Fragen gesellschaftlicher Zugehörigkeit. Die ständigen Verweise auf vermeintliche Regeln im Quartier können - ebenso wie die an autochthone Personen gerichteten Aufforderungen nach (mehr) Integration - in diesem Sinne als Pendant zu den Integrationsaufforderungen gelesen werden, denen die Kinder und J ugendlichen selbst fortwährend ausgesetzt sind und die hinsichtlich ihres Gehalts ähnlich abstrakt bleiben, wie die angeblichen Regeln im Quartier.

Binäre Kategorisierungen in einerseits „Deutsche“ und andererseits „Ausländer“ oder „Kanaken“ manifestieren sich im Sprachgebrauch der Kinder und Jugendlichen. Sie können als Folge einer in der Öffentlichkeit hergestellten „diskursive[n] Spaltung der Gesellschaft in die Kollektive ,Deutsche' und ,Ausländer"“ (Lanz 2007: 173) betrachtet werden. Dieser Annahme liegt die in der kritischen Migrationsforschung weitverbreitete Beobachtung zugrunde, dass „Differenzen und damit verbundene Kategorisierungen [...] [längst] einen ontologischen Status“"(Riegel/Yildiz2011: 168f.) erhalten haben. Die Kinder und J ugendlichen machen in Reaktion darauf 
das Quartier zum Referenzpunkt eigener Logiken von Anerkennung oder Ausschluss bestimmter Personen(-gruppen). Die Voraussetzung dafür sind Gefühle der Zugehörigkeit zum Quartier (place belongingness). Solche Aushandlungsprozesse können als politics of belonging bezeichnet werden, hinter denen sich Machtverhältnisse verbergen, die ihre Wirkung nicht zuletzt auf diskursiver Ebene entfalten (vgl. Antonsich 2010). Dabei handelt es sich um „a discursive resource which constructs, claims, justifies, or resists forms of socio-spatial inclusion/exclusion“ (ebd.: 645). Darüber hinaus gilt: „Every politics of belonging involves two opposite sides: the side that claims belonging and the side that has the power of ,granting' belonging“ (ebd.: 650). In diesem Kontext wird die Zugehörigkeit zu einem Ort gleichbedeutend mit der Zugehörigkeit zu einer Gruppe (vgl. ebd.: 649). In den Worten von Kathleen Mee und Sarah Wright (2009) zeigt sich anhand solcher Situationen, „how belonging is used by people [...] on their own terms. We find a belonging created through active, hybrid, fragile and always contested processes that refuse containment." (Ebd.) Solche Aushandlungsprozesse offenbaren allerdings auch, wie sehr die Rassismuserfahrungen der Kinder und Jugendlichen durch Erfahrungen des Markiert- und Bewertet-Werdens als „Andere“ geprägt sind (vgl. Castro Varela/Mecheril 2016: 16 f.; Hall 2004: 143 ff.). Den meisten von ihnen ist es aufgrund phänotypischer Merkmale nicht möglich, Einfluss auf negative Fremdzuschreibungen zu nehmen. Darum gehören siein den Worten Erving Goffmans (1967: 56) zur Gruppe der „Diskreditierten“. Dass ihre Erzählungen häufig mit Prozessen der (Selbst-)Ethnisierung einhergehen, kann also in erster Linie als Reaktion auf vehemente Zuschreibungen und damit einhergehende Stigmatisierungen als „ethnisch Andere“ verstanden werden. Die Funktion der Selbstethnisierung ist dabei primär die Schaffung eines Gefühls von Zusammengehörigkeit (vgl. Bozay 2012: 122; Ha 2000: 378 ff.). Siekann damitals Schutzreaktion auf Erlebnisse rassistischer Diskriminierung und Ausgrenzung verstanden werden. Aufgrund der gemeinsamen Erfahrung rassistischer Zuschreibungen werden Differenzen bezüglich sozialer und kultureller Herkünfte innerhalb der Peer-Group obsolet. Auf ähnliche Weise dient auch die positive Selbstkennzeichnung als „Kanake“ einer Form der Vergemeinschaftung (vgl. Böder/ Karabulut 2017: 279). Zwar handelt es sich dabei um die Aneignung und Umkehrung einer Begrifflichkeit, die fremdbestimmt und aufgezwungen ist. Durch den Prozess der Aneignung findet jedoch eine Intervention statt. Hinter dieser Form der Aneignung verbirgt sich das Ziel, die rassistische Konnotation des Begriffs zu überschreiben und vollkommen neu zu besetzen (vgl. Ha 2004: 199 ff.). Laut Stuart Hall (2004) können derlei Umwendungen als Teil einer Strategie der Transkodierung verstanden werden: Die Kinder und J ugendlichen nehmen eine „Umkehrung der Bewertungalltagsstruktureller Stereotype“(ebd.: 160) vor, indem sie den Begriff positiv besetzen (vgl. ebd.: $158 \mathrm{ff}$.). Dabei wird diese Selbstbezeichnung und die damit einhergehendeVergemeinschaftungunter den Kindern undJ ugendlichen fortwährend an den Stadtteil rückgekoppelt, in dem sie sich Tag für Tag aufhalten und den sie infolgedessen mit dem Label „Kanakengegend“ versehen. Sonja Preissing (2019a: 241) hält zu solchen Beobachtungen fest: „Das was ihnen gesellschaftlich abgesprochen wird, markieren sie mit der Zugehörigkeit zum Stadtviertel.“ 


\subsection{Positive (Gegen-)Erzählungen}

In etlichen Momenten heben die Kinder und J ugendlichen hervor, wie gerne sieim Quartier am Mehringplatzleben. Besonders gut nachvollziehbarist das anhand der Darstellungen zweierJ ugendlicher, dieim Quartier aufgewachsen sind und in der Anfang 2021 ausgestrahlten rbb-Dokumentation (rbb 2021) zu Wort kommen. Darin spricht die zu diesem Zeitpunkt 21-jährige Leyla von „schöne[n] Erinnerungen“ aus dem Leben im Quartier. Kurz darauf sagt sie: „Der Mehringplatz ist für mich ein Ort vieler Kulturen. Ich finde es schön, dass da so viele verschiedene Nationen auch aufeinandertreffen.“ (rbb 2021) Sie schließt ihren Beitrag mit dem Satz: „Ich würde hier gerne wohnen bleiben. Dasist aufjeden Fall ein Wunsch." (rbb 2021) Der zu diesem Zeitpunkt 20-jährige Deniz vergleicht das gemeinsameAufwachsen im Quartier mit einer Imagination von Dorf, die für ihn äußerst positiv besetzt ist. Dazu sagt er: „Der Vorteil ist, dass wir hier alle zusammenhalten, egal was passiert. Wir haben schlechte Tage, auch gute Tage zusammen mit den J ungs. Wie eine Familie." (rbb 2021) Die etwa viereinhalbminütige Sequenz des Berichts, die den J ugendlichen zugestanden wird, schließt er mit den Worten: „Für mich persönlich schmeckt diese Luft hier viel besser als wenn ich außerhalb der Gegend bin. Wenn wir hier zurückkommen wieder, aus dem Urlaub oder sonst etwas, dann atmen wir einmal tief ein und sagen: ,Endlich haben wir unsere Luft wieder so, unseren Atem. “" (Dabei lacht er) (rbb 2021). DieHervorhebung positiver Eigenschaften des Quartiers, etwa der Vergleich des Zusammenlebens und des gemeinsamen Aufwachsens miteiner idealisierten Vorstellung von Dorf, ist unter den Kindern und J ugendlichen weit verbreitet. Sie betonen das gemeinsame Aufwachsen als unbestreitbare Tatsache, deren positive Konnotation ihnen bei allen stigmatisierenden und abwertenden Darstellungen der Gegend niemand nehmen kann. Dabei stellen sie auch immer wieder heraus, wie viel wohler sie sich im Quartier fühlen als in anderen Teilen der Stadt, in denen sie als Nicht-Angehörige der weißen Mehrheitsgesellschaft stärker auffallen. Solche Erzählungen enthalten Hinweise auf ihre Wertschätzung des Quartiers als Raum, der ihnen einen gewissen Schutz bietet vor den alltäglichen Begegnungen mit Rassismus. Dabei entspricht dieAnnahme, der fehlendesozialeZusammenhalt innerhalb des Stadtteils würde die Identifikation mit dem Quartier belasten - wie es in mehreren wissenschaftlichen Arbeiten sowie im IHEK (vgl. QM 2017: 16) heißt - keineswegs den Darstellungen der Kinder und J ugendlichen. Diese halten dem nahezu vehement entgegen, indem sie in sozialen Medien darauf verweisen, aus „H-Town“[8] zu stammen, ihren Mannschaften bei der Teilnahme an Fußballturnieren Namen wie „H-Town Kickers“ geben, T-Shirts mit der Silhouette der Wohnblocks am Mehringplatz drucken oder ihre Herkunft aus dem Quartier in Rap-Texten verarbeiten. Welche Bedeutung das Viertel für sie hat, zeigte sich auch in Vorbereitung auf den 1. Mai 2019. Mehrere J ugendliche malten ein großes Banner für das MaiFest, das seit einigen J ahren in einem nahegelegenen Kinder- und J ugendzentrum stattfindet, parallel zum bekannten Straßenfest rund um die Kreuzberger Oranienstraße. Es wurde später gut sichtbar an der Außenfassade der Kinder- und J ugendeinrichtung am Mehringplatz befestigt. Darauf stand: „Unser Kiez! Mehr als nur ein Ort, es ist unsere Heimat! Also Händeweg von Kreuzberg!“. 
Abb. 4 Jugendliche befestigen für das MaiFest (2019) ein selbstgemaltes Banner am Zaun eines Kreuzberger Kinderund Jugendzentrums (Quelle: eigene Aufnahme)

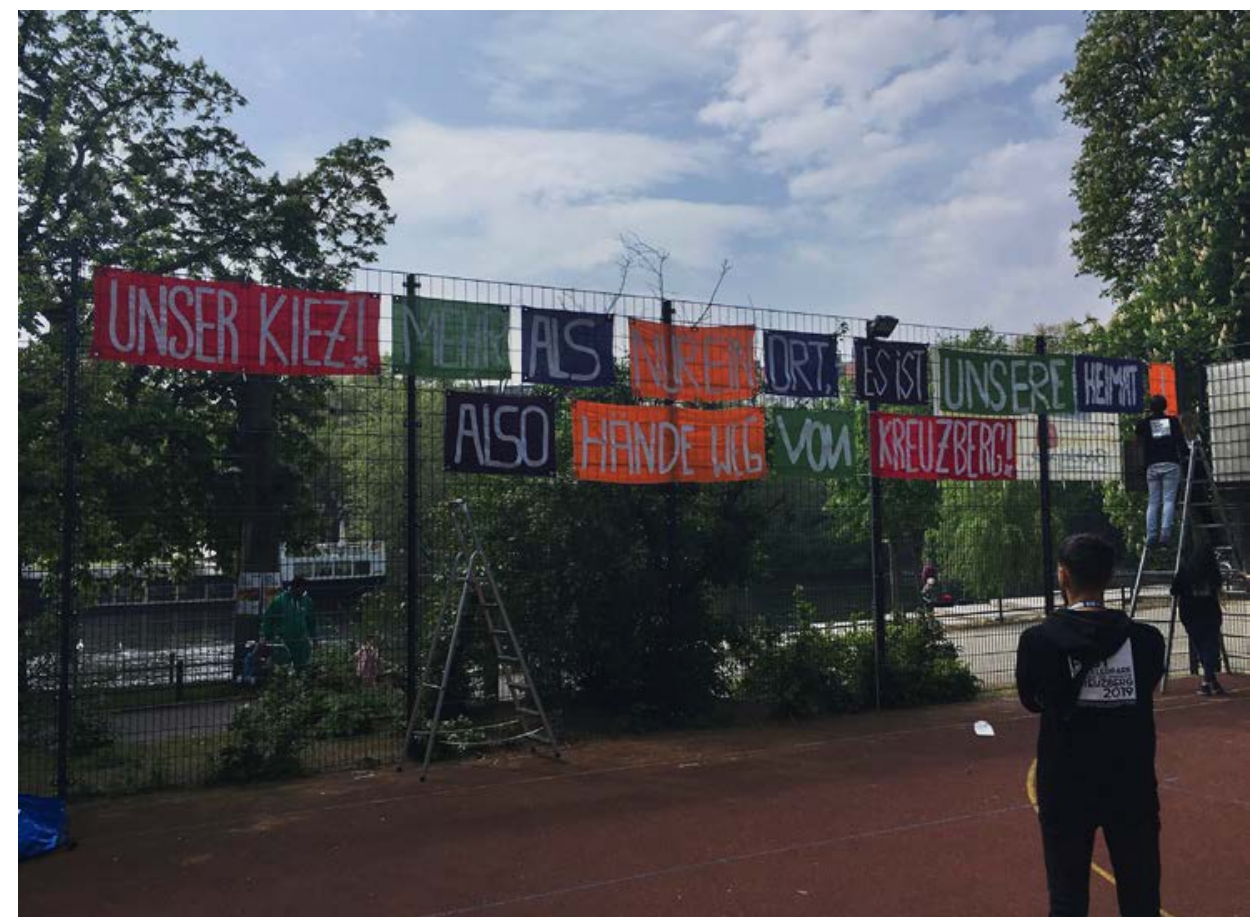

DieForderung „Hände wegvon Kreuzberg“ bezieht sich auf voranschreitende Prozesse der Gentrifizierung und die damit verbundenen Sorgen der J ugendlichen, ausihrem Quartier verdrängt zu werden. Aus Sicht der Kinder und J ugendlichen gilt es die Struktur des Kiezes zu schützen und zu wahren.

Das Beispiel zeigt, wie zutreffend die Annahme ist, es handle sich um „Strategie[n] der Lokalisierung“ (Niedermüller 2000: 124) denen derVersuch zugrunde liege, ,sich mit dem ausgegrenzten Territorium zu identifizieren und dadurch Identität zu territorialisieren, das heißt ,Heimat' zu konstruieren“ (ebd.) und sich im Zuge dessen politisch und gesellschaftlich zu artikulieren. Das Ziel bestehe darin, einen Raum zu schaffen, der Schutz vor Ausgrenzung und Marginalisierung biete (vgl. ebd.). Auf dieseWeise setzen dieKinder und J ugendlichen den abwertenden Darstellungen des Stadtteils etwas entgegen. Begrifflich lässt sich ihre tiefe Verbundenheit zum Quartier als sense of place erfassen. Emotionale Bindungen an einen Ort sind das Resultat subjektiver Erlebnisse und Erinnerungen, in deren Folge diese Orte mit (durchaus unterschiedlichen) Attributen belegt und so zu einem wichtigen Bezugspunkt werden. So können Orte beispielsweise mit einem Gefühl von Heimat oder Sicherheit verbunden werden.[9] Das Place-Konzept umfasst verschiedene räumlicheDimensionen, diein einem wechselseitigen Verhältnis zueinander stehen. Der Fokus meiner Untersuchung liegt auf der symbolischen beziehungsweise diskursiven Ebene, die bei der Konstituierung von place eine zentrale Rolle spielt (vgl. Belina 2017: 108 ff.; Vogelpohl 2014: $61 \mathrm{ff}$.). Das schließt auch medial erzeugte Bilder ein (vgl. Vogelpohl 2014: 63). Zudem verfügen Ortenicht über eindeutige oder einzigartigeund starre Identitäten. Diese sind vielmehräußerst vielfältig. Dementsprechend istauch der sense of place innerhalb einer Gruppe nichts Einheitliches (vgl. Massey 1991: 28 f.). Was places als territoriale Eingrenzung betrifft, gilt für meine Untersuchung: „BBoundaries' may of course benecessary, for the purposes of certain types of studies for instance, but they are not necessary for the conceptualisation of a placeitself."(Ebd.: 29) Bezüglich solcherpositiver (Gegen-)Darstellungen des Quartiers halten Kirkness und Tijé-Dra (2017) fest, dass die Verbundenheit 
zum eigenen Viertel ein wichtiger Schritt sei, um der Macht des Stigmas etwas entgegenzusetzen (vgl. ebd.: 3). Darüber hinaus prangern viele Kinder und Jugendliche regelmäßig an, dass der öffentliche Blick auf das Quartier in keinerlei Verhältnis zu den realen Begebenheiten stehe. Dabei übergehen sie keineswegs, dass das Leben im Stadtteil durchaus mit Schwierigkeiten behaftet sein kann. Es geht ihnen in solchen Momenten nicht primär darum, Probleme im Quartier zu relativieren. Sie fordern lediglich eine differenziertere und weniger dramatisierende Berichterstattung über die Lebensrealitäten und Geschehnisse im Quartier ein.

\subsection{Aneignung des Stigmas}

Mitunter sind es dieselben Kinder und J ugendlichen, die in einem Moment ausschließlich positive Seiten des Quartiers hervorheben und sich im nächsten Moment von ihm distanzieren. Vor allem J ugendliche äußern immer wieder den Wunsch, das Quartier zu verlassen. Das magauf den ersten Blick widersprüchlich erscheinen, muss jedoch als Verweis auf unterschiedliche Strategien im Umgang mit territorialen Stigmatisierungserfahrungen verstanden werden, die sich je nach Situation und Kontext ändern. Dabei werden die in der Öffentlichkeit präsenten negativen Darstellungen durch die Erzählungen der Kinder und J ugendlichen gelegentlich noch weit übertroffen. Es gibt Momente, in denen ein defizitorientierter und ausschließlich problematisierender Blick Überhand gewinnt und in extrem negative Darstellungen gipfelt. Nicht selten kommen dann Metaphern wie „Loch“ oder „Hölle“ zum Einsatz, um die Gegend zu beschreiben. Eine ganz andere Form der (Re-)Produktion stigmatisierender Darstellungen besteht in der bewussten Aneignung des Stigmas. NegativeBilder des Quartiers, verbunden mit Attributen wie „kriminell“ oder „gefährlich“, schreiben sich häufig in die Selbstethnisierungsprozesse der Kinder und J ugendlichen ein. Diese rückbeziehen sie dann wieder auf den Raum, das heißt, sie konstruieren Bilder, die sowohl den gängigen Diskursen über das Quartier entsprechen als auch dem weitverbreiteten ethnisierenden Blick auf J ugendgewalt und -kriminalität. Die Kinder und J ugendlichen machen sich das negative Image des Quartiers zu eigen und nutzen es für ihre Zwecke, etwa um Härte und Macht zu demonstrieren. Auf diese Weise können sie beispielsweise gegenüber Personen, die nicht aus dem Quartier stammen, eine Form von Überlegenheit artikulieren. Erika Schulze und Susanne Spindler (2006) bezeichnen solche Praxen als Taktiken des strategischen Einsatzes des Stigmas (vgl. ebd.: 72 ff.). Moritz Ege (2010) bringt es folgendermaßen auf den Punkt: „Die (relative) Machtlosigkeit in anderen gesellschaftlichen Arenen wird durch Gesten territorialer Dominanz theatralisch kompensiert, überhöht, ästhetisiert." (Ebd.: 62) Dabei spielt auch die Inszenierung von Männlichkeit eine nicht unwesentliche Rolle. Zwar gehen die Kinder und Jugendlichen dadurch ein erhöhtes Risiko ein, bestehende Stereotype zu bestärken und an deren Fortschreibung mitzuwirken, allerdings müssen solche Inszenierungen immer auch als Teil von Bewältigungsstrategien im Umgangmit Fremdzuschreibungen und Marginalisierung betrachtet werden. Die Vorstellung, (junge) muslimische Männer zeichneten sich vor allem durch patriarchale Männlichkeit sowieeinen Hang zu Gewalt und religiösem 
Fundamentalismus aus, ist weit verbreitet (vgl. Spindler 2010: 291). Entsprechende Stereotype existieren also völlig unabhängig von den Selbstpräsentationen und Handlungen der J ugendlichen. Genau das betonen auch die Kinder und J ugendlichen immer wieder. Es sei völlig egal, wie sie sich präsentierten, sie würden ja doch nur als das wahrgenommen, was Andere in ihnen sehen wollen. So gelten J ungen, die ohnehin schon von Rassismus betroffen sind, etwa im Kontext Schule seit geraumer Zeit als die Problemgruppe schlechthin (vgl. Huxel 2011; Spindler 2010: 296 ff.). Dabei ist der Verweis auf ihre vermeintliche Herkunftskultur eine gängige Erklärung für defizitäre Schulleistungen (vgl. Spindler 2006: 297). Aber auch in anderen Kontexten legt sich „Rassismus [...] immer mehr über die jugendlichen Geschlechtskonstruktionen, indem er ihre Männlichkeit als abweichende thematisiert" (Spindler 2010: 304). Katrin Huxel (2011) verweist darauf, dass solcheErfahrungen Auswirkungen auf dieInszenierung von Männlichkeit haben (vgl. ebd.: 94 ff.). Raewyn Connell (2015) spricht diesbezüglich von „protestierende[r] Männlichkeit“ (ebd.: 168). Diese sei „eine marginalisierteForm von Männlichkeit“(ebd.: 173) und entwicklesich „in einer randständigen Klassenlage, wo der für hegemoniale Männlichkeit essentielle Machtanspruch permanent durch wirtschaftliche und kulturelle Schwäche in Frage gestellt wird" (ebd.: 175). Connell unterscheidet hierbei zwischen verschiedenen Formen von Männlichkeit, diezueinander in Beziehung stehen. „Hegemoniale Männlichkeit“ etwa bildet sich über die Ausstattung mit ökonomischen Ressourcen, institutioneller Macht und einem „erfolgreich erhobenen Anspruch auf Autorität“ (ebd.: 131) aus. Dabei definiert sich Männlichkeit stets in Relation zu anderen Männlichkeiten, die entweder inferior oder hegemonial sind. Hegemoniale Männlichkeit darf daher nicht als starr betrachtet werden. Sieist grundsätzlich wandelbar (vgl. Connell 2015). Dazu schreibt Paul Scheibelhofer (2010): „Current hegemonic masculinityis held to be white, heterosexual masculinity" (ebd.: 278, Hervorhebung im Original). Demnach muss die ökonomische Lage, in der sich die meisten der Jugendlichen im Quartier befinden, um spezifische Erfahrungen vergeschlechtlichter Ethnisierung ergänzt werden (Connell 2015). Für sie gilt: „Überall, wo sich die J ugendlichen bewegen, werden ihre Ethnizität und ihr Geschlecht relevant. Ethnisierung und Vergeschlechtlichung sind unmittelbar miteinander verbunden und beeinflussen sich wechselseitig“ (Spindler 2006: 315).[10]

Mit Blick auf sämtliche beschriebene Handlungsweisen bleibt festzuhalten, dass sich die Kinder, und viel mehr noch dieJ ugendlichen in vielen Situationen der Ironie als Stilmittel bedienen. Das führt nicht selten dazu, dass Geschichten oder Inszenierungen im Gelächter aller Beteiligten enden. Das Verständnis von Ironie setzt einen gewissen Konsens an Auffassungen, Betrachtungsweisen und Empfindungen voraus, da sie auf dem Prinzip basiert, gegen diese geteilten Überzeugungen zu verstoßen. Die Ironie darf jedoch nicht über die Ernsthaftigkeit hinwegtäuschen, die sich hinter den Erzählungen verbirgt. Sie kann also ein Mittel sein, das den Kindern und J ugendlichen hilft, mit unterschiedlichen Formen tiefgreifender Diskriminierungs- und Ausgrenzungserfahrungen umzugehen. Erol Yildiz (2017) nennt dies eine „postmigrantische Strategie [...] mit subversiver Wirkung“ (ebd.: 29). 


\section{Fazit}

In den Diskursen, die ich im ersten Teil des Beitrags beschrieben habe, sind vor allem Kinder und J ugendliche eigener Repräsentationen beraubt. Dies muss als besonderer Aspekt ihrer Marginalisierung betrachtet werden. Vor allem für die J üngeren gilt: „Keine andere gesellschaftliche Gruppe ist in ihrer Wahrnehmung so abhängig von räumlichen Zuschreibungen [...] wie Kinder." (Schreiber 2020: 306) Wie aber gehen die jungen Bewohner_innen des Quartiers mit der ständigen Stigmatisierung um? Ich habe drei zentrale Narrative ausgemacht, die in ihrer jeweiligen Verwobenheit vor allem eines deutlich machen: Die Komplexität der Diskriminierungsund Ausgrenzungserfahrungen, die die Kinder und J ugendlichen aus dem Gebiet machen, geht weit über Zuschreibungen auf der räumlichen Maßstabsebene des Quartiers hinaus. Im Kern werden hier gesellschaftliche Auseinandersetzungen auf den Raum übertragen. So wird das Quartier fortwährend zu einem Bezugspunkt der Diskussion um Zugehörigkeit und Ausschluss beziehungsweise um Anerkennungund Nicht-Anerkennung. Das spiegelt sich auch in den Praxen der Kinder und Jugendlichen wider. Neben der von mir beschriebenen diskursiven Umkehr gesamtgesellschaftlicher Verhältnisse heben sie immer wieder die positiven Seiten des Lebens im Quartier hervor. Bei der bewussten Aneignung des Stigmas hingegen findet eine (Re-)Produktion negativer Darstellungen statt. Dadurch darf allerdings nicht der Eindruck entstehen, die Kinder und J ugendlichen seien deshalb selbst verantwortlich für eine Verstetigung stigmatisierender Betrachtungen der Gegend und ihrer Bewohner_innen. Vielmehr sind solche Praxen Reaktionen auf hegemoniale Diskurse. Der Aneignung des Stigmas geht immer die Stigmatisierung voraus. Welche Inszenierungen in der Öffentlichkeit aufgegriffen werden, liegt also nicht in der Hand der Stigmatisierten. Hier macht sich einmal mehr die Machtlosigkeit der Kinder und J ugendlichen im Quartier bemerkbar.

Meine Untersuchung offenbart, dass die Kinder und Jugendlichen Erzählungen produzieren, die ihnen zur Selbstermächtigung dienen. Diese Erzählungen müssen unter Einbezug der Bedingungen gedeutet werden, unter denen sie hervorgebracht werden. Es sind dynamische Erzählungen, die weder als persistent noch als starr betrachtet werden dürfen und die der Situation entsprechend eingesetzt werden. Zudem erfahren diese Erzählungen einen ständigen Austausch innerhalb der Peer-Groups der Kinder und J ugendlichen. Aufgrund dieser Form der Perpetuierung begreifeich sie als Gemeinschaftsnarrative. Auffällig ist, dass die Kinder und Jugendlichen Erfahrungen mit Rassismus sehr viel häufiger benennen, als klassenbasierte Diskriminierungen, auch wenn sich beides häufig überlagert.

Trotz ihres jungen Alters scheinen sich auch die Kinder der Diskurse rund um den Stadtteil und seines schlechten Rufes bewusst zu sein. Ohnehin halten sich dieKinder undJ ugendlichen im Quartier kaum räumlich getrennt voneinander auf. Die Orte, an denen sie innerhalb ihrer Peer-Groups Zeit verbringen, liegen nah beieinander, überschneiden oder wechseln sich ab. Das führt dazu, dass bestimmte Erzählungen innerhalb des Stadtteils weit verbreitet sowie unabhängigvom Alter bekannt sind. Letzten Endes machen Untersuchungen wiediese einmal mehr deutlich, wiewichtiges ist, städtische 
Räumein ihrerjeweiligen Besonderheit zu erfassen sowie Kinder und J ugendliche als Expert_innen ihrer eigenen Lebensrealitäten anzuerkennen. Dies gilt vor allem, wenn sie auf gesellschaftliche Positionen verwiesen werden, die ihnen eigene Repräsentationen massiv erschweren. Kinder undJ ugendliche müssen auch in der Stadtforschung als „aktive Gestalter_ innen von Gesellschaft" (ebd.: 307) anerkannt werden. Entsprechend wichtig ist es, die Perspektiven junger Stadtbewohner_innen aufzuzeigen und ernst zu nehmen.

\section{Endnoten}

[1] Ein besonderes Augenmerksolltedabei auf wirtschaftlicheEntwicklungen, den Abbau des Sozialstaats (vgl. Wacquant 2006: 26 ff.) und die damit einhergehende (Neo-)Liberalisierung von (Stadt-)Politik (vgl. Mayer 2003) gelegt werden - sowie nicht zuletzt auf den Wandel städtischer Ökonomien infolge voranschreitender Tertiärisierung (vgl. Kemper 2018: 7; Farwick 2007: 113 f.).

[2] Ein weiterer Faktor für diese Überschneidungist dieWohndiskriminierung, der migrantisierte Personen auch unabhängig von ihrem Einkommen ausgesetzt sind (vgl. Sarbo/ Wolf 2021: 90; Meksem 2021: 59 f.). Nichtsdestotrotz sind die Hauptursache ethnischer Segregation die sozioökonomischen Verhältnisse (vgl. Farwick 2018: 5).

[3] J ugend wird innerhalb öffentlicher Diskurse ohnehin als defizitär wahrgenommen und ist im Zuge dessen häufig Projektionsfläche für gesellschaftliche Probleme (vgl. Griese 2014: 25; Scherr 2014: 36; Eulenbach/Ecarius 2012: 9). Das gilt in besonderem Maße für migrantisierte Jugendliche, denen innerhalb öffentlicher Diskurse regelmäßig abweichende, gewalttätige und kriminelle Eigenschaften unterstellt werden (vgl. Yildiz 2016: 54 ff.; Griese 2014: 19).

[4] Laut Wacquant geht diese „Auflösung des Ortes“ für die Bewohner_innen der Viertel nicht zuletzt mit einem Gefühl der Unsicherheit einher. Dem kann jedoch beispielsweise die Untersuchung Adefemi Adekunles (2017) entgegengehalten werden, die dieser gemeinsam mit J ugendlichen in London durchführte. Darin widerlegt er die These, dass die Stigmatisierung eines Stadtteils unter den Bewohner_innen unweigerlich in einem Verlust des Sicherheitsgefühls münde.

[5] Allerdings weist Wacquant (2018 [2008]) in Die Verdammten der Stadt darauf hin, dass unter J ugendlichen - im Gegensatz zur restlichen Bevölkerung der stigmatisierten Stadtteile - „die Identifikation mit dem Wohnort [durchaus; Anm. d. A.] übersteigerte Formen“ (ebd.: 295) annehmen kann.

[6] Während die Beiträge im Sammelband von Wacquant/ Slater/ Borges Pereira (2014) vorrangigmarginalisierteStadtteileins Blickfeld nehmen, deren Bewohner_innen stereotypen und abwertenden Darstellungen sowie in einigen Fällen Prozessen (taktisch) herbeigeführter Gentrifizierung und Verdrängung ausgesetzt sind, verdeutlichen die Untersuchungen in Kirkness/ Tijé-Dra (2017), dass bei weitem nicht nur marginalisierte Viertel von Stigmatisierung betroffen sind. Ursachen und (Aus-)Wirkungen territorialer Stigmatisierung sowie Strategien des Umgangs mit dieser können sehr unterschiedlich ausfallen.

[7] Ich habe beide J ugendliche, deren Aussagen aus der rbb-Dokumentation (rbb 2021) ich zitiere, zuvor um ihr Einverständnis gebeten. Anstelle ihrer Namen verwende ich Pseudonyme.

[8] „H-Town“ (englischeAussprache) ist eineBezeichnung für das Quartier am Mehringplatz, die sämtliche Kinder, J ugendliche und junge Erwachsene gebrauchen, mit denen ich zusammenarbeite. Der Buchstabe H leitet sich ab von Hallesches Tor, dem Namen der U-Bahn Station im Quartier. Die Verwendung des Begriffs „H-Town“ reicht laut Erzählungen einiger älterer Anwohner_innen bis in die 1980erJ ahre zurück. Andere oft verwendete Bezeichnungen für das Gebiet sind schlichtweg „Hallesches“ oder „Halle“.

[9] Hierbei ist zu bedenken, dass auch die emotionale Bindung an einen Ort ein Resultat sozialer Verhältnisse und damit keineswegs frei von Machtverhältnissen ist (vgl. Belina 2017: 108). Anne Vogelpohl (2014) hält dazu fest: „Die große Bedeutung von 
Emotionalität und Einzigartigkeit, die in den humanistischen Ansätzen wurzelt, möchte ich [...] zugunsten eher sozialer und politischer Perspektiven relativieren. Denn auch Subjektivitäten und Bewusstsein sind beeinflussbar und teilweise von sozialen, ökonomischen oder politischen Zwängen behindert und eingeschränkt artikulierbar." (Ebd.: 65f.)

[10] Eswärejedoch falsch, davon auszugehen, dass Diskriminierungserfahrungen unweigerlich in dem Versuch münden, Männlichkeit über die Inszenierung von Dominanz und Härte herzustellen. Schon Connell (2015) warntedavor, derartige reduktionistische Schlüssezu ziehen (vgl. ebd.: 129f.). SogenannteProtestmännlichkeiten können sehrunterschiedlich ausgestaltet sein, sie müssen sich also keinesfalls zwangsläufig aus der Übernahme stereotyper Männlichkeitsbilder speisen, sondern können auch mit Selbstdarstellungen einhergehen, dienicht herkömmlichen Rollenverständnissen entsprechen (vgl. ebd.: 171).

\section{Autor_innen}

Nils Zimmer forscht zum Thema Kinder- und Jugendpartizipation im Kontext kultureller Bildungsnetzwerke, weitere Schwerpunkte liegen in der Kritischen Stadt- und Migrationsforschung sowie in der Protest- und Bewegungsforschung.

nils.zimmer@fu-berlin.de

\section{Literatur}

Adekunle, Adefemi (2017): „You have got to represent your ends“. Youth territoriality in London. In: Paul Kirkness / Andreas Tijé-Dra (Hg.), Negative neighbourhood reputation and place attachment. The production and contestation of territorial stigma. London: Routledge, 194-215.

Alisch, Monika (2018): Sozialräumliche Segregation: Ursachen und Folgen. In: Ernst-Ulrich Huster / Jürgen Boeckh / Hildegard Mogge-Grotjahn (Hg.), Handbuch Armut und soziale Ausgrenzung. Wiesbaden: Springer, 503-522.

Antonsich, Marco (2010): Searching for belonging. An analytical framework. In: Geography Compass 4/6, 644-659.

Atteslander, Peter (2010): Methoden der empirischen Sozialforschung. Berlin: Erich Schmidt.

Belina, Bernd (2006): Raum. Überwachung. Kontrolle. Vom staatlichen Zugriff auf städtische Bevölkerung. Münster: Westfälisches Dampfboot.

Belina, Bernd (2017): Raum. Zu den Grundlagen eines historisch-geografischen Materialismus. Münster: Westfälisches Dampfboot.

Böder, Tim / Karabulut, Aylin (2017): „Haftbefehl hat konkret irgendwie einiges für uns geändert". In: Martin Seeliger/ Marc Dietrich (Hg.), Deutscher Gangsta-Rap II. Popkultur als Kampf um Anerkennung und Integration. Bielefeld: transcript, 267-286.

Böhmer, Felix / Holm, Andrej / Jacob, Matthias (2021): Wohnen und Ideologie. In: Andrej Holm (Hg.), Wohnen zwischen Markt, Staat und Gesellschaft. Ein sozialwissenschaftliches Handbuch. Hamburg: VSA, 123-150.

Bozay, Kemal (2012): Probleme und Ursachen der Re-Ethnisierung und Selbstethnisierung im Lehrerzimmer. In: Karim Fereidooni (Hg.), Das interkulturelle Klassenzimmer. Perspektiven neuer deutscher Lehrkräfte auf den Bildungs- und Integrationsdiskurs. Wiesbaden: VS Verlag für Sozialwissenschaften, 117-124.

Breidenstein, Georg / Hirschauer, Stefan / Kalthoff, Herbert / Nieswand, Boris (2015): Ethnografie. Die Praxis der Feldforschung. Konstanz/München: UVK.

Bürkner, Hans-J oachim (2020): Segregation. In: Bernd Belina / Matthias Naumann / Anke Strüver (Hg.), Handbuch Kritische Stadtgeographie. Münster: Westfälisches Dampfboot, 158-163.

Castro Varela, María do Mar / Mecheril, Paul (2016): Die Dämonisierung des Anderen. Rassismuskritik der Gegenwart. Bielefeld: transcript.

Chamakalayil, Lalitha / Gilliéron, Gwendolyn / Günes, Sevda Can / Hill, Miriam / Imširović, Elvisa (2017): Marginalisierte Quartiere? Positionierungen und Deutungen von Bewohner_innen. In: Thomas Geisen / Christine Riegel / Erol Yildiz (Hg.), Migration, 
Stadt und Urbanität. Perspektiven auf die Heterogenität migrantischer Lebenswelten. Wiesbaden: Springer, 157-173.

Connell, Raewyn (2015): Der gemachte Mann: Konstruktion und Krise von Männlichkeiten. Wiesbaden: Springer.

DGSA - Deutsche Gesellschaft für Soziale Arbeit (2020): Forschungsethische Prinzipien und wissenschaftliche Standards für Forschung der Sozialen Arbeit. https:// www.dgsa. de/fileadmin/Dokumente/Ueber_uns/Forschungsethikkodex_DGSA_abgestimmt.pdf (letzter Zugriff am 23.7.2021).

Difu - Deutsches Institutfür Urbanistik(2003): Strategien für dieSozialeStadt. Erfahrungen und Perspektiven - Umsetzung des Bund-Länder-Programms „Stadtteilemit besonderem Entwicklungsbedarf - die soziale Stadt". https://www.bbsr.bund.de/BBSR/DE/FP/ ExWoSt/Studien/2004undFrueher/ProgrammSozialeStadt/DL_Endbericht.pdf? blob=publicationFile\&v=3 (letzter Zugriff am 23.7.2021).

Ege, Moritz (2010): Eine Ästhetik der Territorialität. Postleitzahlen und urbanes Charisma in der Berliner „Straßenmode“. In: Alexa Färber (Hg.), Stoffwechsel Berlin. Urbane Präsenzen und Repräsentationen. Berlin: Panama, 50-65.

Eulenbach, Marcel / Ecarius, Jutta (2012): Zum Systematisierungsdefizit in aktuellen Debatten der Jugendforschung. In: J utta Ecarius / Marcel Eulenbach (Hg.), J ugend und Differenz. Aktuelle Debatten der Jugendforschung. Wiesbaden: Springer, 7-23.

Farwick, Andreas (2007): Soziale Segregation in den Städten - Von der gespaltenen Gesellschaft zur gespaltenen Stadt. In: Detlef Baum (Hg.), Die Stadt in der Sozialen Arbeit. Ein Handbuch für soziale und planende Berufe. Wiesbaden: VS Verlag für Sozialwissenschaften, 111-122.

Farwick, Andreas (2018): Segregation und Integration - ein Gegensatz? http://www.bpb. de/ politik/ innenpolitik/ stadt-und-gesellschaft/ 216880/ segregation-und-integration (letzter Zugriff am 23·7.2021).

Flick, Uwe (2017): Qualitative Sozialforschung. Eine Einführung. Reinbek: Rowohlt.

Franke, Thomas (2014): „Soziale Stadt“ und raumorientiertes Handeln. In: Olaf Schnur (Hg.), Quartiersforschung. Zwischen Theorie und Praxis. Wiesbaden: Springer, 157-173.

Gebhardt, Dirk (2001): „GefährlichefremdeOrte“- Ghetto-Diskursein Berlin und Marseille. In: Ulrich Best / Dirk Gebhardt (Hg.), Ghetto-Diskurse. Geographie der Stigmatisierung in Marseille und Berlin. Potsdam: Universität Potsdam, 3-89.

Geisen, Thomas / Riegel, Christine / Yildiz, Erol (2017): Unterschiedliche Perspektiven auf Migration, Stadt und Urbanität. In: Thomas Geisen / Christine Riegel / Erol Yildiz (Hg.), Migration, Stadt und Urbanität. Perspektiven auf die Heterogenität migrantischer Lebenswelten. Wiesbaden: Springer, 3-16.

Glasze, Georg / Pütz, Robert / Tijé-Dra, Andreas (2014): Stigmatisierung von Stadtvierteln. Einleitung in das Themenheft. Europa Regional 14/2-3, 59-62.

Goffman, Erving (1967): Stigma. Über Techniken der Bewältigung beschädigter Identität. Frankfurt am Main: Suhrkamp.

Griese, Hartmut M. (2014): Jugend - immer noch ein soziales Problem? Persönliche Anmerkungen nach 30 Jahren. In: Axel Groenemeyer / Dagmar Hoffmann (Hg.), Jugend als soziales Problem - soziale Probleme der J ugend? Diagnose, Diskurse und Herausforderungen. Weinheim/ Basel: BeltzJ uventa, 17-28.

Groenemeyer, Axel / Hoffmann, Dagmar (2014): Jugend und soziale Probleme - Einführung in den Band. In: Axel Groenemeyer / Dagmar Hoffmann (Hg.), Jugend als soziales Problem - soziale Probleme der Jugend? Diagnose, Diskurse und Herausforderungen. Weinheim/ Basel: BeltzJ uventa, 11-16.

Ha, Kien Nghi (200o): Ethnizität, Differenz und Hybridität in der Migration: Eine postkoloniale Perspektive. In: Prokla. Zeitschrift für kritische Sozialwissenschaft 30/3, 377-397.

Ha, Kien Nghi (2004): Ethnizität und Migration reloaded. Kulturelle Identität, Differenz und Hybridität im postkolonialen Diskurs. Berlin: Wissenschaftlicher Verlag.

Ha, Noa / Schneider, Andreas (2020): Kritisches Weißsein. In: Bernd Belina / Matthias Naumann / Anke Strüver (Hg.), Handbuch Kritische Stadtgeographie. Münster: Westfälisches Dampfboot, 58-63.

Hall, Stuart (2004): Ideologie, Identität, Repräsentationen. Hamburg: Argument.

Harvey, David (2009 [1973]): Social justice and the city. Athens/London: University of Georgia Press. 
Heinzel, Friederike / Kränzl-Nagl, Renate / Mierendorff, Johanna (2012): Sozialwissenschaftliche Kindheitsforschung - Annäherungen an einen komplexen Forschungsbereich. In: Theo-Web. Zeitschrift für Religionspädagogik 11/ 1, 9-37.

Hill, Marc (2016): Nach der Parallelgesellschaft. NeuePerspektiven auf Stadt und Migration. Bielefeld: transcript.

Holm, Andrej (2010): Gefahren der Aufwertung. https://gentrificationblog.wordpress. com/2010/03/29/ berlin-gefahren-der-aufwertung-interview/ (letzter Zugriff am 23.7.2021).

Huxel, Katrin (2011): Geschlecht und Ethnizität im Feld Schule. In: Bulletin Texte, Zentrum für Transdisziplinäre Geschlechterstudien, Humboldt-Universität zu Berlin 37, 87-101.

Kemper, Jan (2018): Ungleichheit in den Städten. Stadtentwicklung und soziale Ungleichheit. https:// www.bpb.de/ politik/ innenpolitik/ stadt-und-gesellschaft/ 216890/ stadtentwicklung-und-soziale-ungleichheit (letzter Zugriff am 23.7.2021).

Kirkness, Paul (2014): The cités strike back: Restive responses to territorial taint in the French banlieues. In: Environment and Planning A 46, 1281-1296.

Kirkness, Paul / Tijé-Dra, Andreas (2017): Introduction. Exploring urban tainted spaces. In: Paul Kirkness / Andreas Tijé-Dra (Hg.), Negative neighbourhood. Reputation and place attachment. The production and contestation of territorial stigma. London/New York: Routledge, 1-8.

Lanz, Stephan (2002): Mythos europäische Stadt - Fallstricke aktueller Rettungsversuche. In: Wolf-Dietrich Bukow / Erol Yildiz (Hg.), Der Umgang mit der Stadtgesellschaft. Ist die multikulturelle Stadt gescheitert oder wird sie zu einem Erfolgsmodell? Opladen: Leske + Budrich, 63-77.

Lanz, Stephan (2007): Berlin aufgemischt: abendländisch, multikulturell, kosmopolitisch? Die politische Konstruktion einer Einwanderungsstadt. Bielefeld: transcript.

Massey, Doreen (1991): A global sense of place. In: Marxism Today 6, 24-29.

Mayer, Margit (2003): Das Potenzial des Regulationsansatzes für die Analyse städtischer Entwicklungen am Beispiel territorialer Anti-Armutspolitik. In: Ulrich Brand / Werner Raza (Hg.), Fit für den Postfordismus? Theoretisch-politische Perspektiven des Regulationsansatzes. Münster: Westfälisches Dampfboot, 265-280.

Mecheril, Paul / Polat, Ayça (2019): „Richtige“ (sozial-)pädagogische Forschung in „falschen“ Verhältnissen? Von Migrationsforschung als Integrationsforschung zu Migrationsforschung als Kritik. In: Verena Klomann / Norbert Frieters-Reermann / Marianne Genenger-Stricker / Nadine Sylla (Hg.), Forschung im Kontext von Bildung und Migration. Kritische Reflexionen zu Methodik, Denklogiken und Machtverhältnissen in Forschungsprozessen. Wiesbaden: Springer, 47-73.

Mee, Kathleen J . / Wright, Sarah (2009): Geographies of belonging. Why belonging? Why geography? In: Environment and Planning A 41, 772-779.

Meksem, Miriam Zineb (2021): Eine Intersektionale Perspektive auf das Wohnen und die Wohndiskriminierung. In: Andrej Holm (Hg.), Wohnen zwischen Markt, Staat und Gesellschaft. Ein sozialwissenschaftliches Handbuch. Hamburg: VSA, 49-64.

Niedermüller, Peter (2000): Öffentlichkeit und Urban Underclass. In: Martin Wentz (Hg.), Die kompakte Stadt. Frankfurt am Main/ New York: Campus, 119-125.

Ottersbach, Markus (2003): Die Marginalisierung städtischer Quartiere in Deutschland als theoretische und praktische Herausforderung. In: Aus Politik und Zeitgeschichte B 28/2003, 432-439.

Ottersbach, Markus (2009): J ugendliche in marginalisierten Quartieren Deutschlands. In: Markus Ottersbach / Thomas Zitzmann (Hg.), J ugendliche im Abseits. Zur Situation in französischen und deutschen marginalisierten Quartieren. Wiesbaden: VS Verlag für Sozialwissenschaften, 51-76.

Preissing, Sonja (2019a): Jugend am Rande der Stadt. Eine vergleichende Studie zu Marginalisierung und Raumaneignung in Deutschland und Frankreich. Wiesbaden: Springer.

Preissing, Sonja (2019b): Zur Forschung am Rande der Stadt: Herausforderungen, Ambivalenzen und Perspektiven. In: Verena Klomann / Norbert Frieters-Reermann / Marianne Genenger-Stricker / Nadine Sylla (Hg.), Forschung im Kontext von Bildung und Migration. Kritische Reflexionen zu Methodik, Denklogiken und Machtverhältnissen in Forschungsprozessen. Wiesbaden: Springer, 143-152.

QM - Quartiersmanagement am Mehringplatz (2017): Integriertes Handlungsund Entwicklungskonzept 2017-2019 mit Jahresbilanz seit 2015. http://www. qm-mehringplatz.info/fileadmin/ user_upload/IHEK_Mehringplatz_2017_2019.pdf (letzter Zugriff am 23.7.2021). 
rbb - Mehringplatz. Das Tor zur Friedrichstraße (2021): Rundfunk Berlin-Brandenburg (rbb), Heimatjournal, 6.2.2021, 19:00, 26:16. https:// www.rbb-online.de/ heimatjournal/ videos/mehringplatz---das-tor-zur-friedrichstrasse.html (letzter Zugriff am 23.7.2021).

Reimann, Bettina / Schuleri-Hartje, Ulla-Kristina (2005): Integration von Migrantinnen und Migranten im Stadtteil. In: Soziale Stadt info 17, 2-8.

Riegel, Christine / Geisen, Thomas (Hg.) (2010): Jugend, Zugehörigkeit und Migration. Subjektpositionierung im Kontext von Jugendkultur, Ethnizitäts- und Geschlechterkonstruktionen. Wiesbaden: VS Verlag für Sozialwissenschaften.

Riegel, Christine / Yildiz, Erol (2011): Jugendliche mit Migrationshintergrund. Akteure eigener Lebenswirklichkeit oder determinierte Andere? In: Axel Pohl / Barbara Stauber / Andreas Walther (Hg.), Jugend als Akteurin sozialen Wandels. Weiheim/München: J uventa, 163-181.

Rinn, Moritz (2018): Ein Urbanismus der Ungleichheit. In: sublurban. zeitschrift für kritische stadtforschung, 6/1, 9-28.

Ronneberger, Klaus / Tsianos, Vassilis S. (2009): Panische Räume. Das Ghetto und die „Parallelgesellschaft“. In: Sabine Hess / Jana Binder / J ohannes Moser (Hg.), No integration?! Kulturwissenschaftliche Beiträge zur Integrationsdebatte in Europa. Bielefeld: transcript, 137-152.

Sarbo, Bafta / Wolf, Lukas (2021): Politische Ökonomie des Wohnens. In: Andrej Holm (Hg.), Wohnen zwischen Markt, Staat und Gesellschaft. Ein sozialwissenschaftliches Handbuch. Hamburg: VSA, 83-96.

Scheibelhofer, Paul (2010): A question of honour? Masculinities and positionalities of boys with Turkish background in Vienna. In: Christine Riegel / Thomas Geisen (Hg.), J ugend, Zugehörigkeit und Migration. Subjektpositionierung im Kontext von Jugendkultur, Ethnizitäts- und Geschlechterkonstruktionen. Wiesbaden: VS Verlag für Sozialwissenschaften, 275-290.

Scherr, Albert (2014): J ugend als soziale Kategorie. Oder: Warum J ugend keine Gruppe und auch kein soziales Problem ist. In: Axel Groenemeyer / Dagmar Hoffmann (Hg.), Jugend als soziales Problem - soziale Probleme der J ugend? Diagnosen, Diskurse und Herausforderungen. Weinheim/ Basel: BeltzJ uventa, 29-49.

Schreiber, Verena (2020): Kindheit. In: Bernd Belina / Matthias Naumann / Anke Strüver (Hg.), Handbuch Kritische Stadtgeographie. Münster: Westfälisches Dampfboot, 306-312.

Schulze, Erika (2010): „Und ich fühl mich als Kölner, speziell als Nippeser“. Lokale Verortung als widersprüchlicher Prozess. In: Christine Riegel / Thomas Geisen (Hg.), Jugend, Zugehörigkeit und Migration. Subjektpositionierung im Kontext von Jugendkultur, Ethnizitäts- und Geschlechterkonstruktionen. Wiesbaden: VS Verlag für Sozialwissenschaften, 99-112.

Schulze, Erika/ Spindler, Susanne(2006): „,...dann wird man direktalsasozial abgestempelt.“ - Vom Stigma und seinen Folgen. In: Wolf-Dietrich Bukow / Markus Ottersbach / Elisabeth Tuider / Erol Yildiz (Hg.), Biographische Konstruktionen im multikulturellen Bildungsprozess. Wiesbaden: VS Verlag für Sozialwissenschaften, 63-81.

Slater, Tom / Anderson, Ntsiki (2012): The reputational ghetto: Territorial stigmatisation in St Paul's, Bristol. In: Transactions of the Institute of British Geographers (New Series) 37/4, 530-546.

Spindler, Susanne (2006): Corpus delicti. Männlichkeit, Rassismus und Kriminalisierung im Alltagjugendlicher Migranten. Münster: Unrast.

Spindler, Susanne (2010): Eine andere Seite männlicher Gewalt, Männlichkeit und Herkunft als Orientierung und Falle. In: Christine Riegel / Thomas Geisen (Hg.), J ugend, Zugehörigkeit und Migration. Subjektpositionierung im Kontext von Jugendkultur, Ethnizitäts- und Geschlechterkonstruktionen. Wiesbaden: VS Verlag für Sozialwissenschaften, 291-308.

Sylla, Nadine / Frieters-Reermann, Norbert / Genenger-Stricker, Marianne / Klomann, Verena (2019): Forschungspraktische Reflexionen: Eine Einführung. In: Verena Klomann / Norbert Frieters-Reermann / Marianne Genenger-Stricker / Nadine Sylla (Hg.), Forschung im Kontext von Bildung und Migration. Kritische Reflexionen zu Methodik, Denklogiken und Machtverhältnissen in Forschungsprozessen. Wiesbaden: Springer, 89-98.

Terkessidis, Mark (2004): Die Banalität des Rassismus. Migranten zweiter Generation entwickeln eine neue Perspektive. Bielefeld: transcript.

Vogelpohl, Anne (2014): Stadt der Quartiere? Das Place-Konzept und die Idee von urbanen Dörfern. In: Olaf Schnur (Hg.), Quartiersforschung. Zwischen Theorie und Praxis. Wiesbaden: Springer, 59-76. 
Wacquant, Loïc (2006): Das J anusgesicht des Ghettos und andere Essays. Basel: Birkhäuser - Verlag für Architektur und Gütersloh/Berlin: Bauverlag.

Wacquant, Loïc (2007): Territoriale Stigmatisierung im Zeitalter fortgeschrittener Marginalität. In: Das Argument 271 (49/3), 399-409.

Wacquaint, Loïc (2018 [2008]): Die Verdammten der Stadt. Eine vergleichende Soziologie fortgeschrittener Marginalität. Wiesbaden: Springer.

Wacquant, Loïc / Slater, Tom / Borges Pereira, Virgílio (2014): Territorial stigmatization in action. In: Environment and Planning A 46, 1270-1280.

Walther, Uwe-J ens / Güntner, Simon (2007): Soziale Stadtpolitik in Deutschland. Das Programm „Soziale Stadt“. In: Detlef Baum (Hg.), Die Stadt in der sozialen Arbeit. Ein Handbuch fürsozialeundplanendeBerufe. Wiesbaden:VSVerlagfürSozialwissenschaften, 389-400.

Weber, Florian Daniel (2013): Soziale Stadt - Politique de la Ville - Politische Logiken. (Re-)Produktion kultureller Differenzierung in quartiersbezogenen Stadtpolitiken in Deutschland und Frankreich. Wiesbaden: Springer.

Wilkens, J ulien / Klier, Sabine (2018): Trinker, J unkies und Dreck. Der Mehringplatz ist der größte Schandfleck der Hauptstadt. In: B.Z. online, 20.8.2018. https://www.bz-berlin. $\mathrm{de} /$ berlin/friedrichshain-kreuzberg/der-mehringplatz-ist-der-groesste-schandfleck-derhauptstadt (letzter Zugriff am 27.9.2021).

Yildiz, Erol (2017): Postmigrantische Perspektiven auf Migration, Stadt und Urbanität. In: Thoma Geisen / Christine Riegel / Erol Yildiz (Hg.), Migration, Stadt und Urbanität. Perspektiven auf die Heterogenität migrantischer Lebenswelten. Wiesbaden: Springer, 19-33.

Yildiz, Miriam (2016): Hybride Alltagswelten. Lebensstrategien und Diskriminierungserfahrungen Jugendlicher der 2. und 3. Generation aus Migrationsfamilien. Bielefeld: transcript.

Zimmer-Hegmann, Ralf (2014): Zwischen Stigmatisierung und positiver Imageentwicklung - Soziale Stadt als Instrument der Quartiersentwicklung. In: Europa Regional 14/2-3, 120-134.

\section{Community narratives among children and youth in marginalized neighborhoods. Strategies in dealing with stigmatizing discourses at Mehringplatz in Berlin Kreuzberg}

For many years, the Mehringplatz neighborhood in Berlin-Kreuzberg has been subject to immense stigmatization. The discourses about the district arecharacterized by a (re-)production of racist and discriminatory images, ignoring social inequalities and structural disadvantages as results of social power relations. Especially children and young people of the district are deprived of their own representations. Hence, they have hardly any possibility to break up the prefabricated images and the violence inherent to them. This articleasks, which strategies thechildren and teenagers develop in reaction to theseimages and how they themselves perceivethisimportant space. This text is based on the results of an ethnographic study. 